\title{
Castoriadis et l'imaginaire radical : Une confrontation avec la phénoménologie
}

Par NICOLAS POIRIER

Université de Paris Nanterre

Résumé L'objet de cet article est d'examiner ce qui rapproche la pensée de l'imaginaire radical développée par Castoriadis et la phénoménologie dans sa tentative pour thématiser et expliciter la question de l'imagination, tout en indiquant les motifs pour lesquels ces deux approches ne se recoupent nullement, voire s'opposent sur certains points. L'idée sous-jacente à mon argumentation est qu'en arrivant à confronter sur le terrain de l'imagination Castoriadis et certains penseurs qui se situent dans la filiation phénoménologique, on parvient à saisir ce qui peut poser problème avec les positions que défend Castoriadis sur la question : le fait de manquer peut-être le rôle hybride que joue l'imagination, qui pour être créatrice, n'est pas nécessairement aussi originaire que le pense Castoriadis. Et si l'imagination, loin d'être une source première, n'était pas avant tout le lieu d'un passage, l'espace des migrations entre le même et l'autre, qui rend ces deux termes inséparables et comme indiscernables?

\section{Introduction}

L'objet de cette contribution est de confronter les conceptions de Castoriadis concernant l'imagination avec celles défendues par un certain nombre de penseurs appartenant à la tradition phénoménologique, qu'ils en soient à l'origine (Husserl et à sa suite Heidegger), ou qu'ils se situent dans leur filiation plus ou moins directe (Sartre principalement, qui a consacré deux livres importants à cette question). Je vais dans un premier temps faire ressortir ce qui constitue l'originalité de la position de Castoriadis par rapport à ce que la philosophie « héritée » a pensé sous le terme d'imagination, puis je montrerai 
dans un second moment ce qui distingue l'imaginaire radical chez Castoriadis des différentes formes de l'imagination telles qu'elles ont pu être élaborées chez Husserl et Heidegger, et retravaillées à leur suite par Sartre. J'adopterai pour finir une perspective davantage critique afin de mettre en exergue les problèmes posés par cette figure de l'imaginaire radical, en m'efforçant de pointer un certain nombre d'apories sur lesquelles celle-ci semble déboucher.

\section{L'imaginaire radical selon Castoriadis}

S'il y a, d'après Castoriadis, un fait philosophique remarquable, c'est que l'imagination n'ait jamais été pensée comme telle ${ }^{1}$, c'est-à-dire traitée telle une faculté positive, une puissance ou un pouvoir de. On devrait d'ailleurs plutôt dire de l'imagination qu'elle n'aura été justement pensée que comme puissance - le terme devant s'entendre ici au sens dépréciateur d'inachèvement et d'incomplétude (l'être-en-puissance n'est jamais véritablement ni totalement puisqu'il contient un ensemble indéfini de virtualités) - et c'est précisément avec cette conception négative de la puissance que Castoriadis cherche à rompre, en montrant que la puissance n'est pas seulement manque d'être, mais que cette déficience ontologique est une condition positive, en ce sens qu'elle ouvre à une création possible. Son lien constitutif avec les idées d'invention et de création ayant été totalement oblitéré, l'imagination a été ainsi rabaissée au rang de faculté secondaire, au mieux auxiliaire pour la connaissance comme chez Descartes ou Leibniz, au pire comme chez Platon source d'illusion. C'est ainsi qu'au livre VI de La République, Platon définit l'imagination comme la faculté la plus basse dans la hiérarchie des modes de connaissance, en soulignant le caractère tout à fait équivoque d'une telle faculté : car si l'imagination nous permet de représenter les objets en leur absence et constitue à ce titre un auxiliaire utile à la connaissance de l'être, elle est aussi une matrice d'images fantaisistes, c'est-à-dire une source de fantasmes trompeurs qui nous fait prendre nos rêves pour la réalité ; d'où l'ambivalence de l'imagination pour Platon : celle-ci nous pousse, dans le meilleur des cas, à opérer des conjectures sur ce qui est appréhendé, par exemple lorsque nous ne sommes pas sûrs de la véracité des images perçues,

${ }^{1}$ C. Castoriadis, "La découverte de l'imagination», 1978, dans Domaines de l'homme, Paris, Seuil, «Empreintes », 1986, p. 327-331, ainsi que «Imagination, imaginaire, réflexion », 1997, dans Fait et à faire, Paris, Seuil, « La couleur des idées », 1997, p. 227-244. 
et qu'ayant un doute quant à la réalité de ce qui nous apparait, nous préférons suspendre notre jugement plutôt qu'être induits en erreur ; mais l'imagination peut aussi très bien (et c'est ce qui se produit le plus souvent selon Platon) nous faire prendre les images pour la réalité, et nous emprisonner dans une illusion d'autant plus forte que nous avons la certitude d'être en possession de la vérité.

La philosophie aurait certes, d'après Castoriadis, ménagé une place à l'imagination créatrice, en lui reconnaissant une fonction primordiale dans le domaine de l'art, mais c'était pour mieux souligner sa subordination à la sphère du supra-sensible. Il est d'ailleurs absolument remarquable, à suivre ce dernier, que l'art soit la seule dimension où Kant reconnaît le rôle originaire de l'imagination en tant que génie ${ }^{1}$. Kant dépasse sur ce point la tradition philosophique en reconnaissant un mode d'être originairement créateur où sont littéralement produites des formes inédites au travers de l'invention de règles nouvelles ${ }^{2}$ : alors que la théorie esthétique " classique » caractérisait le beau selon des critères objectifs (la perfection, la simplicité, l'harmonie, l'unité) et assignait à l'art le but d'imiter un modèle idéal, la théorie kantienne du génie - «disposition innée de l'esprit par l'intermédiaire de laquelle l'esprit donne à l'art ses règles $\rangle^{3}$ — définit l'œuvre d'art comme une création première à l'image de rien. Le problème est que Kant ne parvient à penser cette création qu'en la subordonnant à la sphère éthique supra-sensible : c'est ainsi que la "tâche » du génie ne consiste pas tant à créer des formes nouvelles qu'à présenter dans l'intuition sensible les idées de la raison, la beauté devenant le symbole du bien moral ${ }^{4}$.

Que l'on sorte ainsi de la perspective rationaliste classique en faisant de l'imagination une faculté magique qui transfigure à ce point le réel qu'elle nous ouvre à un envers du monde, comme dans le cas du surréalisme ${ }^{5}$, ne change rien au problème : qu'elle serve à l'incarnation du Bien ou qu'elle soit une ivresse créatrice, qu'on lui attribue la mission de représenter le «supra-sensible» ou qu'on défende son caractère gratuit et arbitraire, l'imagination n'est jamais pensée pour elle-même : elle reste toujours au service d'autre chose. C'est donc à l'imagination au sens de la faculté de

${ }^{1}$ C. Castoriadis, "La polis grecque et la création de la démocratie », 1982, dans Domaines de l'homme, op. cit., p. 277.

${ }^{2}$ E. Kant, Critique de la faculté de juger, $\$ 46$.

${ }^{3}$ Ibid.

${ }^{4}$ Ibid., § 59. Voir sur ce point C. Castoriadis, « La découverte de l'imagination », op. cit., p. 328.

5 Voir le «Manifeste du surréalisme» de 1924, A. Breton, Manifestes du surréalisme, Paris, Pauvert, 1962 et 1979, rééd. « Folio/Essais », 2001, p. 13-21. 
représenter un objet (qu'il soit réel ou fictif) que la philosophie s'est le plus souvent référée - soit l'imagination conçue en tant que reproduction ou combinatoire d'éléments pré-donnés : une imagination en définitive seconde (elle ne crée pas l'objet mais se borne à le produire après-coup : reproduction) et secondaire (elle ne figure pas au titre des facultés supérieures de l'esprit, mais se contente de rendre présentable le matériau sensible fourni par la perception $)^{1}$. La philosophie aurait donc toujours défini l'imagination, en naviguant entre deux écueils : ou bien l'imagination relève de l'infrapensable, ses objets restent indéterminés et son être privation de détermination, en tout cas déficient quant à ce qu'il détermine ; ou bien l'imagination tient du supra-pensable, son objet est alors indéterminable, non par défaut d'être, mais au contraire par excès, et la source de son être reste une transcendance inaccessible à toute détermination ${ }^{2}$.

Castoriadis va chercher à repenser l'imagination comme une source de création première, en montrant que la distinction apparemment fondatrice pour l'ontologie héritée du « réel » et de l'« imaginaire » n'est en fait qu'une opposition dérivée de cette imagination radicale. L'imagination sous sa forme radicale doit être prise comme synonyme d'imaginaire premier, au sens où cet imaginaire crée non seulement des images au sens trivial du terme, mais plus généralement des formes, et par là il faut entendre aussi bien le langage que des types génériques (représentations abstraites), soit l'ensemble des significations au travers desquelles le monde «prend forme » pour l'homme. L'imagination renvoie à une réalité substantielle, ce n'est pas un simple adjectif dénotant une qualité ou qualifiant un certain type d'objet : ainsi les significations imaginaires sociales ne constituent pas de simples représentations mentales formées par les membres d'une collectivité mais sont les attributs des sociétés en tant que telles, ce qu'il conviendrait de nommer dans la perspective de Montesquieu ou de Hegel «l'Esprit objectif $»^{3}$. L'imagination radicale forme ce à partir de quoi surgissent les schèmes et les figures qui conditionnent toute représentation et toute pensée possibles. Les oppositions structurantes de la pensée philosophique (réel/fictif, intelligible/sensible, rationnel/irrationnel...) en sont toutes dérivées. Pour l'exprimer en un vocabulaire moderne, on pourrait dire que l'imagination

\footnotetext{
${ }^{1}$ C. Castoriadis, « La découverte de l'imagination », op. cit., p. 328.

${ }^{2}$ Ibid., p. 327.

3 Voir sur ce point O. Fressard, "L’idée de création social-historique », dans Imaginaire et création historique, dir. Philippe Caumières, Sophie Klimis et Laurent Van Eynde, Bruxelles, Publications des Facultés universitaires Saint-Louis, 2006, p. 96.
} 
radicale forme la «condition transcendantale » du pensable et du représentable : au fond, sans cette présentation première, ou plus exactement sans cette création première, il n'y aurait rien à proprement parler pour l'homme, aucune image ou représentation des choses (que cette représentation soit « sensible» ou bien «intelligible ») ${ }^{1}$. L'imaginaire radical prend deux formes : l'imaginaire social (ou société instituante), qui crée les significations s'incarnant dans le complexe des institutions de la société (l'imagination à l'œuvre dans la société et l'histoire) ; l'imagination radicale de la psyché (l'imagination à l'œuvre dans le psychisme humain individué) ${ }^{2}$.

\section{Castoriadis et la phénoménologie}

Initié à la philosophie par la lecture de Max Weber et des penseurs néokantiens, Castoriadis a entretenu des rapports assez étroits avec la phénoménologie, notamment Husserl, auteur auquel il a consacré dans les années 1950 un grand nombre de textes restés inédits. La relation de Castoriadis à la phénoménologie est ambivalente : s'il critique de façon très féroce Sartre, autant sur le plan philosophique que politique, et dans une moindre mesure Heidegger, il se montre plus ouvert à l'encontre de Husserl, et surtout de Merleau-Ponty, auquel il a consacré deux articles assez élogieux ${ }^{3}$. Il a par ailleurs été assez proche de Ricoeur, qui dirigea la thèse de doctorat qu'il ne soutiendra par ailleurs jamais. Quant à la pensée de Levinas, elle semble lui être restée complètement étrangère. Sa conception de l'autonomie peut sembler en partie redevable à la démarche phénoménologique, puisqu'il s'agit de penser sous ce terme l'activité d'un sujet originairement ouvert à l'altérité, et prenant conscience de lui-même dans un mouvement d'ouverture au monde, qui n'est pas à l'origine son monde propre mais un monde commun. L'autonomie, dira Castoriadis dans L'institution imaginaire de la société, est cette forme d'agir, cette praxis, qui vise la liberté d'autrui par le

\footnotetext{
${ }^{1}$ C. Castoriadis, « La découverte de l'imagination », op. cit., p. 352-353, ainsi que «Anthropologie, philosophie, politique », 1989, dans La montée de l'insignifiance, Paris, Seuil, « La couleur des idées », 1996, p. 110-111.

${ }^{2}$ C. Castoriadis, «Imagination, Imaginaire et réflexion », op. cit., p. 228, ainsi que L'institution imaginaire de la société, Paris, Seuil, «Esprit», 1975, rééd. «Points/Essais », 2006 , p. 533.

${ }^{3}$ C. Castoriadis, « Merleau-Ponty et le poids de l'héritage ontologique », 1976-1977, dans Fait et à faire, op. cit., p. 157-195; « Le dicible et l'indicible », 1971, dans Les Carrefours du labyrinthe, Paris, Seuil, « Empreintes », 1978, rééd. « Points/Essais », 1998, p. 161-189.
} 
moyen même de cette liberté ${ }^{1}$. Elle ne désigne ni un état achevé, ni un idéal, mais un projet «pratico-poïétique » qui s'exprime sous deux formes : d'une part, un projet éthique de transformation de soi et du rapport de soi à l'autre, d'autre part un projet politique de transformation de la société. Ce qui ferait plus généralement tout l'intérêt de la phénoménologie réside dans la recherche d'un dépassement des antinomies que Castoriadis juge constitutives de la philosophie traditionnelle, principalement celle mettant aux prises un sujet qui doit reconnaître, dans un objet pourtant complètement séparé de lui, une forme sur laquelle son mode de structuration cognitif peut avoir prise. Ce qu'il finira par reprocher à la phénoménologie, notamment husserlienne, c'est de ne pas avoir réussi à accomplir le projet consistant à sortir des impasses de la "pensée contemplative », suivant ses propres termes, en raison principalement de son incapacité à traiter du problème autrement que dans les termes de l'égologie d'obédience cartésienne.

Pour ce qui concerne plus précisément le problème de l'imagination, Husserl s'est employé à cerner le mode d'être qui est celui de l'acte par lequel la conscience cherche à viser l'objet en tant qu'image, et c'est dans son sillage que Sartre va faire ressortir la structure intentionnelle de l'image. Ayant pris assez rapidement ses distances avec la conceptualité husserlienne, Heidegger a pour sa part repensé la question de l'imagination hors des cadres psychologiques et cognitifs qui sont, semble-t-il, encore ceux de Husserl, dans le but de montrer que ce qui se joue pour l'essentiel avec l'imagination dépasse de loin les seuls domaines de la psychologie et de la théorie de la connaissance.

\section{Castoriadis confronté à la phénoménologie : points de convergence}

Ce qui peut apparaître comme un premier point de convergence entre Castoriadis et la perspective phénoménologique se situe au niveau du rapport entre imagination et liberté : en se référant au caractère intentionnel de la conscience, Sartre montre que l'imagination est une activité de la conscience consistant dans une certaine manière pour elle de viser un objet ${ }^{2}$. L'intérêt d'une telle conception tient à ce qu'elle permet de sortir du psychologisme inhérent aux philosophies classiques de la connaissance, dont le défaut consistait à assimiler l'objet avec sa représentation, faisant du coup perdre

\footnotetext{
${ }^{1}$ C. Castoriadis, L'institution imaginaire de la société, op. cit., p. 112-113.

2 J.-P. Sartre, L'imaginaire, Paris, Gallimard, 1940, rééd. « Folio/Essais », 1986, p. 21.
} 
toute consistance à la réalité ${ }^{1}$. L'erreur selon Sartre serait de croire que l'image se trouve dans la conscience (elle serait en quelque sorte un état de conscience), et que l'objet représenté soit lui-même comme immanent à l'image. Il ne faut pas en effet confondre l'objet de la conscience et le contenu de cette même conscience qu'il transcende : l'objet visé par la conscience constitue le corrélat noématique de la conscience mais n'appartient pas à celle-ci à titre de contenu ${ }^{2}$. Il s'ensuit que l'image n'est pas un contenu de conscience, mais désigne, comme l'affirme Sartre, le rapport que la conscience entretient avec l'objet ${ }^{3}$. L'imagination constitue donc suivant cette logique un acte positionnel de la conscience qui se manifeste dans son intentionnalité néantisante en constituant un objet comme inexistant; ainsi imaginer Pierre, pour reprendre l'exemple récurrent de Sartre, c'est poser Pierre sur le mode de l'absence, c'est le néantiser et le présenter ainsi à sa conscience en tant qu'absent ${ }^{4}$.

En faisant de l'imagination une façon pour la conscience de viser un objet, la phénoménologie sartrienne identifie donc dans l'imagination un pouvoir, celui de constituer l'image, qui n'est pas une donnée factuelle (au sens où celle-ci serait une chose objectivement reconnaissable), mais la résultante d'un acte de visée intentionnelle: en ce sens, l'image est une création de la conscience constituante ${ }^{5}$. Le point commun avec Castoriadis consiste précisément dans le refus de réduire l'imagination à une simple impression. Imaginer quelque chose, ce n'est pas se contenter d'enregistrer passivement des données, dont la force serait telle qu'elles s'imprimeraient dans l'esprit de l'extérieur. L'imagination radicale n'est pour Castoriadis en rien passive : elle désigne chez lui cette puissance de création, ou si l'on préfère cette capacité à faire être, capacité qui ne tire sa substance d'aucun fondement préexistant ${ }^{6}$, ce qui n'est pas sans faire écho chez Sartre à l'entière liberté pour la conscience de faire surgir, d'un être en-soi caractérisé par son inertie, un monde de significations. Toute la différence vient de ce que Sartre s'en tient à l'imagination en tant que faculté psychologique individuelle, et ne considère jamais celle-ci comme une puissance de création sociale irréductible à la conscience.

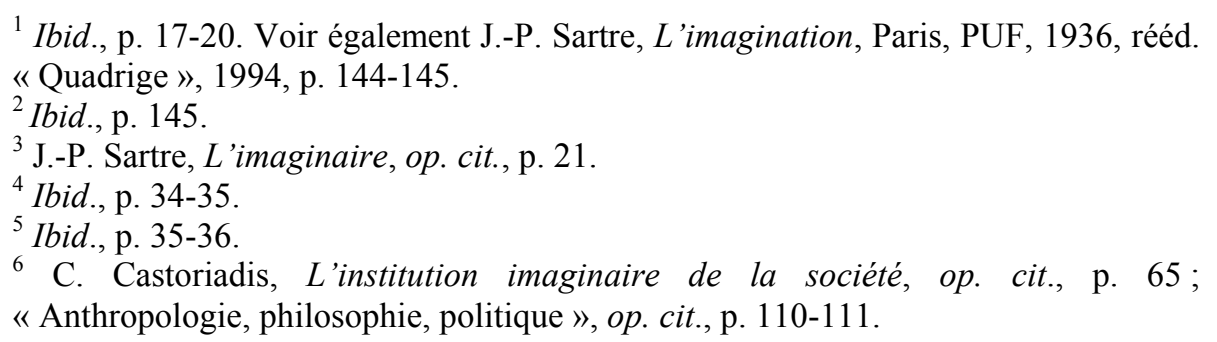


Ce n'est d'ailleurs pas anodin si Sartre voit dans l'imagination, non un simple pouvoir s'ajoutant à sa capacité perceptive, mais le mode essentiel par lequel la conscience réalise son être-au-monde précisément en tant que liberté. La fonction néantisante de la conscience par laquelle celle-ci dépasse son « être-jeté », pour reprendre le vocabulaire de Heidegger, et transcende donc sa facticité, ne peut se manifester qu'à travers l'action d'imaginer. Ainsi que l'affirme Sartre, toute situation dans laquelle apparaît la conscience est intrinsèquement porteuse d'imaginaire, en ce sens que celle-ci se présente comme une façon de dépasser le donné. Il s'avère en effet toujours possible de nier la réalité du monde tel que la conscience le découvre, de le poser, en totalité ou dans une ou plusieurs de ses parties, comme irréel, en imaginant qu'une autre réalité est possible, et en s'employant à la transformer selon la perspective imaginée ${ }^{1}$. Ainsi, penser avec Sartre que l'homme est absolument libre, c'est signifier par là que c'est à lui de donner sens à sa situation, en choisissant de s'y résigner ou au contraire de la refuser. Ce qui veut donc dire que l'homme possède la capacité de nier les déterminations extérieures qui tendent à le figer dans une situation réifiée et de poser sur un mode irréel, autrement dit d'imaginer, une situation où il assumera, sans sombrer dans la mauvaise foi, son entière liberté. C'est également parce que les hommes sont doués, d'après Castoriadis, d'une imagination radicale qu'ils peuvent se montrer capables de poser des déterminations différentes de celles qui leur sont présentées comme les seules légitimes, de faire surgir d'autres possibles en référence auxquels ils peuvent chercher à transformer leurs conditions d'existence.

Cette mise en suspens des déterminations données est ce qui va introduire du jeu au sein de situations dont la configuration apparaissait comme définitive et qui semblait donc exclure de son champ toute autre perspective. Ceci revient à créer de nouveaux espaces rendant possible une exploration utopique de la réalité, où les évidences normatives sont contestées dans leur prétention à épuiser le champ des possibles. L'exercice critique de l'imagination implique donc un travail d'indétermination mettant en valeur le fait que d'autres manières de déterminer les choses sont possibles : le caractère légitime de ce qui est simplement parce qu'il existe se voit ainsi mis radicalement en question.

Si l'imagination nous met au contact d'objets, en tant qu'ils sont visés sur un certain mode par la conscience intentionnelle, elle le fait d'une

${ }^{1}$ J.-P. Sartre, L'imaginaire, op. cit., p. 358-361. Voir également sur ce point F. Noudelmann, Sartre : l'incarnation imaginaire, Paris, L'Harmattan, « Ouverture philosophique », 1996, p. 26-28. 
manière bien spécifique, puisqu'elle ne statue pas sur l'existence effective, donc sur la réalité, des objets envisagés. Il en résulte une double perspective : la première, c'est l'insistance sur la dimension irréelle des objets visés par l'acte d'imagination, et c'est dans cette direction que Sartre semble s'orienter, puisque pour faire ressortir le contenu de la vie imaginaire, la première chose qu'il met en évidence est le caractère magique de l'acte d'imagination, qui en reste au stade de l'incantation s'évertuant à faire apparaitre l'objet par la seule grâce de la pensée, d'où son côté enfantin caractérisé précisément par le refus de tenir compte du principe de réalité ${ }^{1}$. La visée intentionnelle de la réalité sur le mode de l'imagination révèle bien souvent une tentative pour fuir la réalité ou s'y confronter en faisant preuve de mauvaise foi. La seconde perspective, c'est la mise en suspens de tout jugement portant sur la réalité des choses. L'essentiel ici ne consiste pas à poser l'irréalité des objets auxquels on pense, mais à ne pas tenir compte du fait que ces objets imaginés n'existent pas et ne sont donc pas réels - les objets de l'imagination sont considérés comme tels abstraction faite du problème de leur existence. C'est la voie suivie par Husserl, qui dans certains de ses aspects, rappelle la réflexion de Castoriadis concernant l'imaginaire instituant.

La démarche adoptée par Husserl dans les Idées directrices pour une phénoménologie accorde en effet une place centrale à l'imagination: il semble donc légitime de rapprocher le penseur allemand de Castoriadis. Le problème est abordé par Husserl dans le cadre de la réflexion qu'il mène pour réussir à comprendre comment la conscience donatrice originaire peut parvenir à la saisie des essences. Ainsi, dans le premier chapitre de la section inaugurale où celui-ci s'emploie à distinguer le fait et l'essence, Husserl soutient l'idée que la connaissance de l'essence est absolument indépendante de la connaissance portant sur les faits. C'est pourquoi il est, d'après lui, tout à fait possible d'emprunter diverses voies afin de parvenir à la saisie originaire de l'essence, puisqu'il n'y a aucune raison de privilégier le mode de perception matérielle à partir du moment où ce n'est pas le fait qui est spécifiquement visé : la voie des intuitions empiriques correspondantes bien entendu, mais également celle des intuitions sans rapport avec l'expérience et par lesquelles il est impossible de conclure à l'existence de l'objet, ce que Husserl désigne par l'expression «intuitions purement fictives $»^{2}$. L'imagination constituerait de cette manière une façon pour la conscience

\footnotetext{
${ }^{1}$ J.-P. Sartre, L'imaginaire, op. cit., p. 239.

2 E. Husserl, Idées directrices pour une phénoménologie, trad. P. Ricoeur, Paris, Gallimard, 1950, rééd. Gallimard, «Tel », 2005, p. 24-25.
} 
donatrice de saisir l'essence d'une chose ${ }^{1}$ : Husserl va jusqu'à accorder une fonction privilégiée à l'imagination dans le processus de connaissance. D'après ce dernier, la présentification de l'essence par le biais de l'imagination peut en effet s'avérer d'une clarté parfaite et donc rendre à elle seule possible une compréhension adéquate de l'essence ${ }^{2}$.

La saisie des essences dans le domaine des mathématiques constitue à cet égard le cas paradigmatique permettant de comprendre les raisons pour lesquelles l'imagination libre a une position privilégiée par rapport aux seules perceptions pour ce qui concerne la visée des essences. Contrairement à un préjugé assez répandu, l'imagination en mathématiques n'a pas qu'une fonction illustrative ; elle a également un rôle exploratoire, qui occupe une place très importante dans ce type de raisonnement ${ }^{3}$. L'image ne doit donc pas être conçue comme ce qui, faute de mieux, permet de fournir un support matériel graphique et visuel à des recherches et démonstrations abstraites. Elle forme bien davantage ce à partir de quoi le mathématicien explore des possibilités nouvelles: l'intuition imaginative produit des figures et des schémas que le mathématicien peut faire varier dans le but de parcourir l'ensemble des configurations possibles en se donnant le droit de créer, quasiment à l'infini, de nouvelles figures: l'objectif d'un tel travail de reconfiguration ou de recomposition est de parvenir à saisir, au gré des variations eidétiques, la forme unitaire de l'essence dans sa nécessité, soit ce qui de l'essence résiste à l'effort pour en produire des variations, ou si l'on préfère, des figures contingentes ${ }^{4}$. C'est pourquoi la fiction constitue d'après Husserl l'élément vital de la phénoménologie et plus largement des sciences eidétiques, elle constitue même selon lui la source où puise la connaissance des essences, de ce qu'elles ont d'intemporel et de nécessaire ${ }^{5}$.

L'imagination doit être ici conçue dans la perspective d'une mise en suspens de ce qui se donne au sujet sur un mode ne faisant pas question. Cette prise de distance par rapport à la question de la réalité effective va dès lors rendre possible une transformation de la réalité donnée en un objet d'interrogation : par le refus de statuer sur ce qui s'offre à nous, comme si c'était naturel, l'imagination se donne ainsi la capacité de poser des possibilités qui n'avaient jusque-là jamais été envisagées. Or ce qui caractérise, d'après Castoriadis, l'imaginaire sous sa forme instituante, c'est de pouvoir

\footnotetext{
${ }^{1}$ Ibid., p. 223-227.

${ }^{2}$ Ibid., p. 223.

${ }^{3}$ Sur ce point, voir, dans le présent volume, le texte de David Rabouin.

${ }^{4}$ E. Husserl, Idées directrices pour une phénoménologie, op. cit., p. 225-226.

${ }^{5}$ Ibid., p. 227.
} 
mettre en question la totalité de ce qu'une société présente comme institué, c'est-à-dire comme valant de par le seul fait de son existence ${ }^{1}$. Il ne s'agit pas uniquement d'une critique des institutions telles qu'elles existent à un moment donné dans l'histoire, et du fait qu'on pourrait en créer de nouvelles ou même simplement envisager la possibilité qu'elles puissent fonctionner autrement, mais de la mise en question du fait même de son institution, et plus généralement du fait de l'institution comme telle. Cet exercice de mise en suspens de la valeur du monde institué doit déboucher sur la reconnaissance que l'institution d'une société ne tire pas son être d'une instance transcendante, par exemple d'origine divine, qu'elle n'a donc rien d'incontestable: sa légitimité ne vaut donc pas de manière absolue sous prétexte qu'elle serait fondée en une entité divine détentrice de la vérité ${ }^{2}$.

Mais des penseurs venus de la phénoménologie, hormis MerleauPonty, c'est peut-être de Heidegger, pour ce qui concerne en tout cas la reconnaissance du lien originaire entre imagination et temporalité, que Castoriadis semble, sur ce point tout au moins, le plus proche. Ce que voulait montrer Heidegger dans son livre de 1929, Kant et le problème de la métaphysique, c'est qu'il existe une dimension plus originaire que celle relative au sujet et à l'objet - les diverses formes de leur mise en rapport constituant l'objet traditionnel de la philosophie de la connaissance. Ce lieu est la temporalité, et c'est précisément ce que révèle l'analyse heideggerienne de l'imagination transcendantale ${ }^{3}$. Ce que Kant aurait apporté de novateur dans sa conception du schématisme, avec toutefois beaucoup d'ambiguïtés, c'est de réussir à concevoir le sujet transcendantal comme immergé dans le temps ${ }^{4}$, même si d'après Heidegger, Kant aurait marqué un net recul dans la seconde édition de la Critique de la raison pure. Toute la question est de savoir si le sujet empirique ne peut parvenir à la connaissance de la réalité que parce qu'il est doublé par un sujet transcendantal qui lui fournit les catégories dotées d'un caractère universel et nécessaire, ou bien s'il est dès l'origine inséré dans une temporalité constitutive. D'après l'interprétation qu'en fournit Heidegger, l'imagination transcendantale, en rendant possible

\footnotetext{
${ }^{1}$ Voir à ce sujet C. Castoriadis, «Imaginaire politique grec et moderne», dans $L a$ montée de l'insignifiance, op. cit., p. 161.

${ }^{2}$ Voir par exemple C. Castoriadis, La société bureaucratique (Introduction de 1972), Paris, Christian Bourgois, 1990, p. 52, ainsi que «Institution de la société et religion », 1982, dans Domaines de l'homme, op. cit., p. 383.

${ }^{3}$ M. Heidegger, Kant et le problème de la métaphysique, trad. A. De Waelhens et W. Biemel, Paris, Gallimard, 1953, rééd. Gallimard, «Tel», notamment p. 242 et p. 251.

${ }^{4}$ Ibid., p. 246-250.
} 
la temporalisation des catégories, unirait de manière originaire, via le schématisme, ce qui chez Kant restait en définitive séparé et ne se trouvait réuni que de façon très équivoque ${ }^{1}$. L'imagination constitue en quelque sorte ce qui ouvre le sujet à la temporalité originaire et rend compte de son historicité intrinsèque. Du coup, bien que la problématique de l'ontologie fondamentale ne puisse être confondue avec la réflexion menée par Castoriadis sur le lien entre temps et création ${ }^{2}$, il y a chez les deux philosophes une tentative, certes menée par des voies différentes, pour voir dans l'imagination, transcendantale ou radicale, la source même de l'historicité. C'est ainsi que pour Castoriadis, l'imagination, conçue de manière radicale comme puissance de création et émergence du nouveau, devait rendre pensable un projet d'émancipation politique, par la considération finalement occultée chez Marx que ce sont les hommes eux-mêmes qui créent leur propre histoire.

\section{Castoriadis confronté à la phénoménologie : points de divergence}

Ces rapprochements de Castoriadis avec la phénoménologie restent sans doute comparativement moins importants que ce qui les sépare, pour s'en tenir notamment à Sartre. Car si l'auto-institution du social relève bel et bien de la liberté des hommes, on aurait tort de n'y voir, à suivre Castoriadis, qu'une forme contingente sans plus : que la position première des significations imaginaires ne puisse se justifier de manière ultime ne signifie pas que ce processus relève d'un acte gratuit ${ }^{3}$. La liberté dont parle Sartre serait de ce point de vue analogue à la liberté d'indifférence dont parle Descartes, qui consiste à choisir presque à l'aveugle ou par hasard entre deux possibilités ${ }^{4}$.

Par ailleurs, l'assimilation sartrienne de la liberté à un pouvoir de néantisation, dont l'imagination constituerait une forme privilégiée, se situe dans un cadre égologique des plus classiques ${ }^{5}$. Le principal problème est que

1 J. Rivelaygues, Leçons de métaphysique allemande, tome II. Kant, Heidegger, Habermas, Paris, Grasset et Fasquelle, « Collège de philosophie », 1992, p. 342-356, plus particulièrement p. 350-353.

${ }^{2}$ C. Castoriadis, « Temps et création », 1988, dans Le monde morcelé, Paris, Seuil, « La couleur des idées », 1990, p. 247-278.

${ }^{3}$ C. Castoriadis, «Institution de la société et religion », op. cit., p. 383-384.

${ }^{4}$ Sur la critique de Sartre par Castoriadis, voir C. Castoriadis, « De la monade à l'autonomie », dans Fait et à faire, op. cit., 1991, p. 86-87.

${ }^{5}$ Voir sur ce point N. Poirier, L'ontologie politique de Castoriadis. Création et institution, Paris, Payot, « Critique de la politique », p. 101-102 et p. 114-116. 
Sartre pense l'imagination précisément à partir de la conscience souveraine s'arrogeant le privilège de constituer le sens à partir de son seul rayonnement, sans prendre en compte l'insertion du sujet dans un champ social et historique qu'il n'a pas constitué et qui lui préexiste en-deçà du pouvoir signifiant de sa conscience, travaillé qui plus est par l'inconscient ${ }^{1}$. Bien que l'on trouve aussi des éléments qui vont dans ce sens chez Sartre - de ce point de vue Castoriadis manque-t-il sans doute de nuances -, il faut toutefois se demander si la Critique de la raison dialectique ne constitue pas avant tout une réactivation de l'ontologie phénoménologique, ne prenant pas en compte la dimension du social, mise en œuvre par Sartre dans L'être et le néant.

Les positions de Sartre qui identifient imagination et néantisation seraient, à suivre Castoriadis, exemplaires de la tendance la plus idéaliste de la philosophie moderne, consistant à refuser de penser la créativité contenue dans le faire et l'agir propres à l'homme autrement qu'en termes de négation, oubliant que le moment d'objectivation, c'est-à-dire d'extériorisation, est un moment nécessaire dans le processus de création, faute de quoi la création se limite à n'être qu'un surgissement évanescent dont ne subsiste aucune trace. Cette forme de pensée, qui s'exprime de façon paradigmatique chez Kojève, dans le cadre de l'interprétation qu'il fournit de la Phénoménologie de l'esprit, part du postulat très contestable que l'objectivation ou l'extériorisation constituent une forme d'aliénation à travers laquelle le sujet perd l'essentiel de sa souveraineté. Ce type de pensée est en effet fondé sur le modèle d'une opposition absolue entre l'histoire, qui relève d'une pure action du sujet régi par le principe de la négation, et la nature, qui au contraire s'épuise à parcourir sans cesse le même cercle sans parvenir à en tirer quelque chose de neuf ${ }^{2}$. Dans ces conditions, seule l'activité qui consiste à nier le donné naturel, en transformant par des moyens techniques ce qui n'est là qu'en vertu d'une nécessité aveugle, peut être assimilée à la liberté authentique qui n'est pas simple pouvoir-être conformément à ses dispositions initiales, mais avant tout négation déterminée apte à faire surgir de l'être-en-soi (ou ce qui revient au même de l'identité pure et simple) un univers de sens ${ }^{3}$. Or en séparant de manière aussi radicale le sujet et l'objet, l'hyper-subjectivisme de Sartre ou de Kojève finit par invalider la possibilité de la création. La difficulté tient en effet à ce qu'il semble très problématique

${ }_{1}^{1}$ C. Castoriadis, L'institution imaginaire de la société, op. cit., p. 155-161.

2 A. Kojève, Introduction à la lecture de Hegel, Paris, Gallimard, 1947, rééd. Gallimard, « Tel », 1980, p. 486.

${ }^{3}$ Ibid., p. 494. 
de penser la création simplement comme une puissance de surgissement, qui plus est conçue comme pure négativité. Car si la création implique nécessairement un moment de mise en question radicale, elle doit également intégrer le moment de l'objectivation, où le produit se sépare du producteur et acquiert une existence indépendante.

Il est d'ailleurs caractéristique que Sartre voit dans la création une sorte de contradiction ontologique, "poursuite vaine ", comme il l'affirme dans les Cahiers pour une morale, « de l'En-soi-Pour-soi » ${ }^{1}$. De la même manière que le déterminisme objectiviste, le subjectivisme radical se montrerait en fait incapable de penser la possibilité de la création. La confusion vient, d'après Castoriadis, qu'on caractérise trop rapidement comme aliénation ce qui est d'abord pensable en tant qu'extériorisation et expression de soi, et dans une telle optique, la logique voudrait que l'on tienne la création artistique pour une forme d'aliénation ${ }^{2}$. L'activité du sujet qui s'objective hors de lui-même est certes toujours confrontée à quelque chose de déjà constitué, qui en forme la condition, et à ce titre, la source de possibilités et d'impossibilités ${ }^{3}$. Mais cette activité n'est pas négatrice au sens où Kojève et Sartre entendent ce terme, c'est-à-dire qu'elle ne consiste pas à s'extirper du donné, mais à y faire surgir de nouveaux contenus en créant des possibilités inédites; elle n'est pas non plus aliénante car, si la position de possibles nouveaux conditionne de façon irréversible l'action future, en ce sens que la réalisation de telle possibilité renvoie au néant les possibles concurrents, il est de toute façon dans la logique de l'action que celle-ci se situe sur un terrain qu'elle n'aura pas entièrement délimité et qui s'impose à elle comme une force contraignante: l'histoire ne peut jamais avancer qu'en prenant appui sur son propre passé ${ }^{4}$. Non seulement on ne doit pas voir dans le langage un mode d'être aliénant qui rendrait le sujet parlant étranger à ce qu'il cherche à signifier, le sens de ses dires fuyant à travers les mots qui objectivent et donc figent, voire emprisonnent, sa pensée mais on ne peut non plus faire de l'action une structure en soi aliénante, puisque fondée sur un moment d'objectivation de soi. ${ }^{5}$. Le problème de l'autonomie fait immédiatement surgir la question du rapport à l'autre, mais ce lien qui n'est

\footnotetext{
${ }^{1}$ J.-P. Sartre, Cahiers pour une morale, Paris, Gallimard, « Bibliothèque de philosophie », 1983, p. 156.

${ }^{2}$ C. Castoriadis, «Création et aliénation», 1960, dans Histoire et création. Textes philosophiques inédits 1945-1967, Paris, Seuil, «La couleur des idées », 2009, p. 105.

${ }^{3}$ Ibid., p. 104.

${ }^{4}$ Ibid., p. 104-105.

${ }^{5}$ Ibid., p. 103-104.
} 
pas uniquement intersubjectif puisqu'il est avant tout social et historique, contient le déterminant positif du sujet et rend pensable le champ de déploiement constitutif de son action. Ce n'est donc pas dans l'objectivation que se situe l'aliénation, mais dans l'impossibilité pour le sujet de réfléchir son rapport à l'altérité ${ }^{1}$.

Par ailleurs, si Sartre reconnaît à la subjectivité imageante un rôle majeur comme pôle d'intentionnalité constitutif de l'être-au-monde en tant que liberté, c'est pour dans le même temps souligner la pauvreté essentielle de l'image ${ }^{2}$ : en raison de son peu de matérialité, celle-ci ne peut jouer qu'un rôle illustratif ou métaphorique au sein du processus de connaissance. Imaginer un objet, c'est certes le constituer en tant qu'image : en ce sens l'image est bien la résultante d'une dynamique tangible ayant un effet déterminé. Mais c'est également, à suivre Sartre, anéantir l'objet mis en image, puisqu'il ne subsiste que sous la forme d'un analogon, et l'isoler de tout contexte perceptif. La pauvreté inhérente à l'image tiendrait ainsi d'après Sartre à ce que, à l'inverse de ce qui se produit dans la perception, l'objet visé par l'image ne contient jamais plus que la conscience que l'on s'en forme : alors que la perception ouvre à ce qui déborde du monde des choses - en ce sens il y a dans la perception infiniment plus que ce que nous en voyons - l'imagination se limite aux données que la conscience a constituées en visant l'objet comme image. Ce que veut dire Sartre, c'est qu'un objet ne peut être perçu sans être dans le même mouvement renvoyé à l'infinité des autres objets avec lesquels il entretient une infinité de relations. Rien de tel d'après lui avec l'imagination : l'image se donne d'un bloc, elle ne révèle rien, il n'y a rien à en apprendre, rien à déchiffrer ou à interpréter derrière les apparences ${ }^{3}$.

À l'inverse de ce que pense Sartre, l'une des propriétés de l'imaginaire sous sa forme radicale consiste précisément, d'après Castoriadis, dans cette opération de mise en rapport à l'infini où tout est dans tout. Or le sens, dans ce qu'il a de partageable, ne peut être constitué par un sujet conscient qu'à la condition que celui-ci renonce justement à ce désir de signification totale et de résorption du vide ou de la séparation dans la fusion avec l'autre ${ }^{4}$. Ce qui peut donc poser problème avec l'imagination radicale, ce n'est pas tant l'isolement et l'incapacité d'une mise en relation constitutive de tout processus cognitif que la difficulté d'y introduire des interstices permettant de sortir

${ }_{1}^{1}$ C. Castoriadis, L'institution imaginaire de la société, op. cit., p. 150-158.

2 J.-P. Sartre, L'imaginaire, op. cit., p. 26.

${ }^{3}$ Ibid., p. 24-30.

${ }^{4}$ C. Castoriadis, L'institution imaginaire de la société, op. cit., p. 425.

472 
de ce mouvement de renvoi à l'infini. C'est par ailleurs ce qui fait la grande richesse de l'imagination, notamment dans ses formes artistiques : l'imaginaire qui est la source d'une œuvre d'art est d'autant plus riche qu'il ouvre à un horizon interprétatif quasi-infini. L'exemple de la psychanalyse qui s'est élaborée en grande partie sur ce principe l'atteste également: le processus d'interprétation des rêves, et plus généralement le principe de la libreassociation, sont construits sur le principe suivant lequel la mise en rapport d'un terme à ce à quoi il renvoie implique sa mise en relation avec la totalité des autres termes ${ }^{1}$.

Le second reproche que Castoriadis pourrait adresser à Sartre, et qui vaut plus largement pour Husserl et la phénoménologie en général, est l'impossibilité de penser la société comme un pôle originaire de créativité, au sein duquel le sujet est inséré dès sa naissance. Le social ne peut en effet, dans une démarche comme la phénoménologie fondée sur le principe de la subjectivité fondatrice, faire autrement qu'identifier la société à l'intersubjectivité, ou plus précisément, au réseau des relations que les sujets entretiennent les uns aux autres. Or l'assimilation de la société à l'intersubjectivité rend impossible, à suivre Castoriadis, la reconnaissance du social comme pôle de créativité anonyme et intrinsèquement historique: l'existence est certes toujours existence à plusieurs, mais elle est aussi et avant tout existence sociale et historique. L'intersubjectivité forme bien en ce sens la dimension constitutive de ce que nous sommes en nous différenciant les uns des autres ; elle constitue en quelque sorte la chair concrète des relations humaines et forme à ce titre un moment incontournable de l'expérience humaine. Néanmoins, les sujets qui entrent en relation les uns avec les autres ne sont pas n'importe quels sujets, ils sont les sujets institués par telle société à tel moment de son histoire, et comme tels, participent du type anthropologique produit par l'institution première de la société : pensée de manière fondamentale, l'existence à plusieurs constitue donc elle-même la résultante d'un type donné d'institution social-historique, même si pour chaque individu, elle est toujours vécue comme relation subjective à l'autre. On ne peut donc, à suivre Castoriadis, définir le social-historique uniquement comme l'addition indéfinie des réseaux intersubjectifs (bien qu'il soit aussi cela), ni même comme leur résultante ${ }^{2}$. Le social-historique doit se penser

\footnotetext{
${ }^{1}$ Ibid., p. 405.

${ }^{2}$ Ibid., p. 160-161. Conformément à ce que suggère Alievtina Hervy, on pourrait peut-être nuancer cette critique de Castoriadis, si l'on se rapporte aux développements de Husserl dans la Krisis et, particulièrement, au thème de la " générativité ", qui entend précisément rendre compte de la formation socio-historique du sens, de la
} 
comme un collectif anonyme instituant, et c'est uniquement à partir de l'imaginaire radical que l'on peut concevoir la réalité conjointe de la société et de l'histoire - ce que Castoriadis appelle le "social-historique ${ }^{1}$. L'imaginaire social est le pôle créateur à l'origine non seulement du complexe d'institutions qui caractérise une société mais plus fondamentalement de la forme même de l'institution. Cet imaginaire social ne peut être pensé que comme la capacité créatrice du collectif anonyme mis en œuvre à chaque fois, sous une forme bien spécifique, que des hommes sont rassemblés ${ }^{2}$.

\section{Critique de l'imaginaire radical}

Le motif qui orientera notre conclusion est qu'en raison de son caractère extrêmement massif - c'est d'ailleurs ce qui fait sa force - la conception castoriadienne de l'imagination manque peut-être d'une certaine finesse : au fond, si la pensée de Castoriadis met bien en évidence une dimension essentielle de l'imagination à laquelle la métaphysique traditionnelle s'était montrée aveugle, il semble pour elle plus difficile de parvenir à saisir ce qui se joue dans le fonctionnement concret de l'imagination, et dans ce que celleci rend possible, notamment en ce qui concerne le rapport à l'altérité et sa compréhension. Mon hypothèse est qu'en raison du caractère par trop radical de sa conception de l'imaginaire, au sens où il s'agit pour lui de repenser les choses à la racine, Castoriadis n'aura sans doute pas réussi à saisir véritablement la créativité immanente au champ social-historique de la fin du $20^{\mathrm{e}}$ siècle, qu'il caractérisait de façon très unilatérale comme "montée de l'insignifiance ». À trop vouloir remonter à la source ou à l'origine, Castoriadis aura peut-être fini par manquer de sensibilité aux détails des choses et à leur singularité.

On l'a remarqué précédemment, c'est en tant que puissance de créativité originaire que Castoriadis conçoit l'imagination dans sa radicalité. De ce point de vue, Castoriadis rompt avec la métaphysique traditionnelle, en ce sens qu'il pense l'imagination comme une source créatrice primaire dont la

manière dont il se trouve réactivé, modifié et sédimenté dans les sociétés. Et qui permet d'aborder avec Husserl la problématique de la normalisation qui procède de cette sédimentation préalable.

${ }^{1}$ C. Castoriadis, «Pouvoir, politique, autonomie », 1988, dans Le monde morcelé, op. cit., p. 113.

${ }^{2}$ C. Castoriadis, dans « Anthropologie, philosophie, politique », op. cit., p. 113. 
conceptualité ou la rationalité constituent des modalités secondes : contrairement en effet à Descartes, par exemple, pour qui le pouvoir figuratif de l'imagination est intrinsèquement limité et ne peut égaler la force d'abstraction propre au concept, Castoriadis voit dans l'imaginaire, aussi bien sur le plan de la psyché individuelle que sur celui du social-historique, l'unique puissance ontologique ayant un pouvoir illimité, puisque c'est en référence seulement à cette forme créatrice instituante que l'idée même de limite peut avoir un sens. C'est sans doute parce qu'il insiste sur cette dimension d'activité inhérente à l'être en tant que puissance auto-formatrice que Castoriadis se sépare de la phénoménologie, et c'est à partir de là que l'on peut, en partie au moins, comprendre le sens et la portée de son différend philosophique avec Claude Lefort. Si tous deux conçoivent l'être comme travaillé par un désordre intrinsèque - le chaos (Castoriadis), la division du social (Lefort) - Castoriadis fait ressortir dans cette source originaire une dimension d'activité créatrice là où Lefort insiste davantage sur la passivité qui est ouverture à plutôt qu'ouverture $d e$. Parce qu'il refuse toute réduction de l'imaginaire au reflet, Castoriadis tend à accorder une attention pouvant sembler démesurée à l'aspect créateur de l'imaginaire au détriment de sa dimension réceptive. Il reprochait d'ailleurs à Arendt de privilégier dans la théorie esthétique de Kant la seule dimension du jugement, c'est-à-dire le problème de la réception de l'œuvre d'art par le spectateur, et d'ignorer de la sorte l'imagination créatrice propre au génie artistique, ce qui constituerait pourtant l'apport essentiel de Kant à la question de l'imaginaire ${ }^{1}$. Cette insistance sur l'aspect créateur de l'imagination faisant passer en second ce qui tient en elle de la réceptivité s'inscrit dans la critique faite par Castoriadis de la pensée contemplative, qui accorde sa primauté à la vue et réduit la praxis à ne valoir que de manière subordonnée au regard visant la vérité ${ }^{2}$. Ce privilège du voir sur l'action expliquerait l'impossibilité pour la pensée héritée d'envisager le fait même de la création, qui n'est pas pensable à partir de l'imagination, si l'on choisit de mettre en avant, non la source formatrice, mais la réceptivité, et par conséquent ce qu'elle comporte de passif. Tout se passe en fait comme si Castoriadis

\footnotetext{
${ }^{1}$ C. Castoriadis, « La polis grecque et la question de la démocratie », op. cit., p. 280281. Voir sur ce point J.-C. Poizat, «L'imagination chez Cornelius Castoriadis et Hannah Arendt: le politique entre pouvoir de "création" et pouvoir de "révélation" ", dans Cornelius Castoriadis. Réinventer l'autonomie, dir. Blaise Bachofen, Sion Elbaz et Nicolas Poirier, Paris, éditions du Sandre, 2008, p. 22-23.

${ }^{2}$ C. Castoriadis, notamment «Les antinomies de l'attitude théorique », dans Histoire et création..., op. cit., p. 119. Voir également La société bureaucratique (Introduction de 1972), op. cit., p. 49.
} 
s'intéressait davantage à l'imagination en tant que puissance effective de création qu'à ses résultats tangibles que constituent les images, et par rapport auxquelles effectivement, le sujet est toujours plus ou moins passif.

On peut donc formuler l'hypothèse que c'est parce qu'il se focalise sur ce rôle primordial de l'imagination, la transformant pour ainsi dire en principe fondateur, au détriment de sa fonction médiatrice plus modeste, que Castoriadis aura quelque peu manqué ce rôle intermédiaire de l'imagination, en tant qu'elle se définit dans la tension entre l'universel et le singulier et trace un chemin par où circuler entre le même et l'autre. Un être doté d'un imaginaire radical doit précisément apprendre à faire vivre cette tension entre la propension à penser et agir par soi-même et la tendance à s'abandonner à ce qui vient d'ailleurs. Il semble d'ailleurs définitivement impossible de séparer les images surgissant du flux psychique inhérent au sujet, ce qui atteste chez lui d'un pouvoir actif, et les images qui lui viennent du dehors, et par rapport auxquelles il reste passif. L'imaginaire ne devrait donc pas être conçu comme radical mais davantage sur un mode hybride et comme situé dans un entre-deux : à la fois actif par son pouvoir de réfléchir les images, et passif par sa faculté à être touché par elles, le sujet est absolument seul dans le monde imaginaire qu'il s'est créé, tout en se montrant pourtant apte à partager son monde par sa confrontation à l'épreuve du commun. C'est du coup une phénoménologie de la tension entre lien et séparation qu'une reprise critique de la pensée castoriadienne de l'imagination doit chercher à mettre en œuvre, toute la question revenant à savoir dès lors comment articuler le rapport du même et de l'autre, en évitant le double écueil de l'identification fusionnelle et de l'affirmation de soi démesurée.

\section{Bibliographie}

Breton André, Manifestes du surréalisme, Paris, Pauvert, 1962 et 1979, rééd. « Folio/Essais », 2001.

Castoriadis Cornelius, Les Carrefours du labyrinthe, Paris, Seuil, «Empreintes», 1978, rééd. « Points/Essais », 1998.

—Domaines de l'homme, Paris, Seuil, « Empreintes », 1986.

— Fait et à faire, Paris, Seuil, « La couleur des idées », 1997.

- Histoire et création. Textes philosophiques inédits 1945-1967, Paris, Seuil, « La couleur des idées », 2009.

- L’institution imaginaire de la société, Paris, Seuil, «Esprit», 1975, rééd. « Points/Essais », 2006.

—Le monde morcelé, Paris, Seuil, « La couleur des idées », 1990. 
—La montée de l'insignifiance, Paris, Seuil, « La couleur des idées », 1996.

- La société bureaucratique, Paris, Christian Bourgois, 1990.

Cahiers Castoriadis. Imaginaire et création historique, dir. Philippe Caumières, Sophie Klimis et Laurent Van Eynde, Bruxelles, Publications des Facultés universitaires Saint-Louis, 2006.

Cornelius Castoriadis. Réinventer l'autonomie, dir. Blaise Bachofen, Sion Elbaz et Nicolas Poirier, Paris, éditions du Sandre, 2008.

Heidegger Martin, Kant et le problème de la métaphysique, trad. A. De Waelhens et W. Biemel, Paris, Gallimard, 1953, rééd. Gallimard, « Tel», 1981.

Husserl Edmund, Idées directrices pour une phénoménologie, trad. P. Ricoeur, Paris, Gallimard, 1950, rééd. Gallimard, « Tel », 2005.

Kant Emmanuel, Critique de la faculté de juger.

Kojève Alexandre, Introduction à la lecture de Hegel, Paris, Gallimard, 1947, rééd. Gallimard, « Tel », 1980.

Noudelmann François, Sartre: l'incarnation imaginaire, Paris, L'Harmattan, « Ouverture philosophique », 1996.

Poirier Nicolas, L'ontologie politique de Castoriadis. Création et institution, Paris, Payot, « Critique de la politique », 2011.

Rivelaygues Jacques, Leçons de métaphysique allemande, tome II. Kant, Heidegger, Habermas, Paris, Grasset et Fasquelle, " Collège de philosophie », 1992.

Sartre Jean-Paul Sartre, Cahiers pour une morale, Paris, Gallimard, «Bibliothèque de philosophie », 1983.

—L'imaginaire, Paris, Gallimard, 1940, rééd. « Folio/Essais », 1986.

—L'imagination, Paris, PUF, 1936, rééd. « Quadrige », 1994. 